Itinéraires Itinéraires

Littérature, textes, cultures

\title{
Éthique et mystique scientifique dans Le Principe (2015) de Jérôme Ferrari
}

Ethics and Scientific Mysticism in Le Principe (2015) by Jérôme Ferrari

Isabelle Bernard

\section{(2) OpenEdition}

Journals

Édition électronique

URL : http://journals.openedition.org/itineraires/3704

DOI : $10.4000 /$ itineraires.3704

ISSN : 2427-920X

Éditeur

Pléiade

Référence électronique

Isabelle Bernard, "Éthique et mystique scientifique dans Le Principe (2015) de Jérôme Ferrari », Itinéraires [En ligne], 2017-1 | 2018, mis en ligne le 15 février 2018, consulté le 21 avril 2019. URL http://journals.openedition.org/itineraires/3704; DOI : 10.4000/itineraires.3704

Ce document a été généré automatiquement le 21 avril 2019

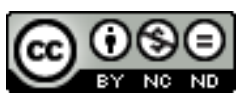

Itinéraires est mis à disposition selon les termes de la licence Creative Commons Attribution - Pas d'Utilisation Commerciale - Pas de Modification 4.0 International. 


\section{Éthique et mystique scientifique dans Le Principe (2015) de Jérôme Ferrari}

Ethics and Scientific Mysticism in Le Principe (2015) by Jérôme Ferrari

Isabelle Bernard

1 Jérôme Ferrari est l'auteur ${ }^{1}$ d'un recueil de nouvelles et de six romans parus chez Actes Sud depuis 2001, notamment du Sermon sur la chute de Rome couronné du prix Goncourt en $2012^{2}$. À la rentrée 2015, il publie Le Principe ${ }^{3}$, un roman qui, tout en dressant un portrait contrasté du pionnier de la physique quantique, Werner Heisenberg (1901-1976), convoque l'histoire européenne du premier demi-siècle. Habile à employer les sermons augustiniens ${ }^{4}$ dans des leçons philosophiques applicables à l'époque moderne et à ses vanités, le narrateur dépeint avec beaucoup de lyrisme (et plus de cinquante ans de recul) la montée du nazisme en Allemagne à partir de 1933, la compromission intellectuelle et les lâchetés des universitaires, les camps de concentration d'une «Europe [...] transformée en équarrissoir [...] en abattoir» $(L P: 99,133)$ et le bombardement de Hiroshima en 1945, dans une longue adresse à Heisenberg. Lui-même se trouve présentement à Dubaï et constate face à cette cité tentaculaire, jaillie du désert avec la même force que l'or noir qui l'irrigue, une sorte de brusque accélération temporelle (153) qui, selon une visée cyclique, transfigure l'état d'alerte écologique actuel en déclin civilisationnel.

2 Ferrari ${ }^{5} s^{\prime}$ inscrit dans un groupe d'auteurs quadragénaires ${ }^{6}$ - héritiers d'une génération qui, elle, connut la Seconde Guerre mondiale puis la décolonisation- qui, pour appréhender le monde d'aujourd'hui, interroge la mémoire individuelle et collective de ses ascendants, et sert autant que se sert de l'histoire. Auteur de $\mathrm{HHhH}$ (2010), Laurent Binet, atteste que les années 1940 se présentent comme un inépuisable réservoir imaginaire : «L'histoire de la Seconde Guerre mondiale est une histoire totale qui, d'une certaine manière, contient toutes les autres, passées et à venir. C'est, potentiellement, la mère (la matrice) de tous les romans » (2011: 85). 
3 L'intérêt que Ferrari porte à la physique moderne s'avère un autre trait saillant de sa fiction biographique ${ }^{7}$ qui entre en résonance avec des romans plus anciens - à commencer par Aleph zéro (2002: 7) - amalgamant en leur sein les paradoxes de la microphysique et, par allusion, citation ou référence, métaphore ou mise en abyme, exploitant le même principe d'incertitude: La Ligne de Sceaux de Daniel Schiff (1982), Monsieur de Jean-Philippe Toussaint (1987), Le Principe d'incertitude de Michel Rio (1992), Les Particules élémentaires de Michel Houellebecq ([1998] 2001) ou encore Le Chat de Schrödinger de Philippe Forest (2013).

$4 \quad$ Nous proposons donc de scruter le fonctionnement intime du Principe afin de dégager les enjeux littéraires de cette œuvre hybride placée sous le signe de l'histoire, de l'épistémologie et d'une méditation sur la transcendance: nous verrons comment les hypothèses et théories quantiques informent le romanesque pour bâtir une écriture ambitieuse et formaliste, à l'intertexte foisonnant, qui dévoile un projet littéraire entre biofiction et autofiction fondé sur un réalisme historique et scientifique prenant en considération une mystique scientifique ayant traversé les âges.

\section{Hypothèses et théories quantiques}

Philosophe de formation, Ferrari bâtit un court roman nourri par un imposant matériel documentaire dont les sources plurielles assurent qu'il n'y a pas de fausse objectivité à l'adresse du lecteur : biographies et autobiographie (celle du physicien s'intitule La Partie et le Tout ${ }^{8}$ ), témoignages écrits, notamment les correspondances de Heisenberg avec d'autres physiciens que Ferrari s'est fait traduire de l'allemand ( $L P: 162)$, enregistrements (ceux de Farm Hall effectués en 1945 - 107-142), et rencontres avec Martin Heisenberg, qui lui a ouvert le fonds personnel de la famille (des photographies sont décrites, notamment celle reproduite sur la jaquette de couverture ${ }^{9}$ ). Ainsi enrichi de données factuelles, Le Principe est à même d'expliquer les avancées de la physique, véritable art, depuis Platon, d'évoquer les mystères de la nature; il pose consécutivement des questionnements fondamentaux sur les implications philosophiques de la physique nouvelle.

Depuis que Max Planck avait découvert le quantum universel d'action, cette funeste constante $h$ qui avait, en quelques années, contaminé les équations de la physique avec la célérité maligne d'un virus impossible à éradiquer, la nature semblait prise de folie : des brisures discrètes fissuraient l'antique continuité des flux d'énergie, la lumière grouillait d'étranges entités granuleuses et, dans le même temps, comme si ce n'était pas suffisant, la matière se mettait à rayonner sauvagement dans un halo fantomatique d'interférences [...] et plus le temps passait, plus il devenait évident que cette épouvantable dualité ne constituait nullement l'exception mais la règle, une règle à laquelle personne ne comprenait quoi que ce soit. (LP:22-23)

6 Féru de sciences, Ferrari s'attache aux progrès de la physique atomique qui, avec la théorie des quanta mise au point dans les années 1920, procède d'une rupture épistémologique radicale. En tentant de modéliser le comportement de l'énergie à l'échelle de l'infiniment petit, les physiciens européens - qui tous, dans la fiction, gravitent autour du protagoniste, leur confrère Heisenberg: Max Planck, Albert Einstein, Louis de Broglie, Wolfgang Pauli, Niels Bohr - ont été à la base de la nouvelle vision de la réalité $^{10}$ : en dépassant les théories de la physique classique, ils furent un jour conduits à bouleverser de fond en comble la vision rationaliste et matérialiste du réel. Cette révolution conceptuelle née "aux limites extrêmes où l'exercice de la pensée devient 
physiquement douloureux » (LP:13), concerne l'élucidation du mystère de la structure de l'atome et de l'organisation des électrons autour du noyau.

7 Le principe qui donne son titre au roman est le principe d'incertitude ${ }^{11}$ énoncé par Heisenberg en 1927 qui régit les relations entre la position et la vitesse d'une particule élémentaire et que Ferrari étend à son art d'écrire ; l'existence de ce principe signifie une limite de la connaissance de la vitesse ou de la position d'une particule si, auparavant, l'on a mesuré de l'une ou de l'autre les coordonnées. Valable pour le couple position et vitesse, le principe l'est également pour le couple temps et énergie. Cette découverte stipule qu'il n'y a pas de trajectoire mais des positions et une vitesse qu'un observateur ne peut jamais mesurer simultanément mais seulement connaître séparément. À l'échelle subatomique $^{12}$, force est de faire le constat simple et désespérant qu'il ne «demeure aucun vestige du monde que le langage des hommes peut décrire» (LP: 12-13). L'interrogation qui sous-tend le roman apparaît donc comme éminemment philosophique et poétique puisqu'elle met en lumière l'inadéquation du langage humain ${ }^{13}$ pour décrire la réalité. L'existence du narrateur que lui-même retrace d'une façon morcelée, elliptique, soulignant des états et des moments de vie calque cette donnée : le roman emprunte sa texture au principe quantique de base puisqu'il confère au Je une identité rhizome, selon l'expression deleuzienne. Le langage est mis au premier plan de l'interrogation portée par l'hypothèse du physicien puisqu'il se définit comme inapte à appréhender la « splendeur abstraite de la matière éternelle » (13), inapte à exprimer l'instabilité fondamentale des univers physiques et biologiques. C'est «entre une métaphore et le silence » (11), dans «le refuge des formes mathématiques » que « la raison a sa demeure » (29).

La composition quadripartite du roman reprend ces données essentielles dans la description des particules subatomiques. Ainsi, "positions ", "vitesse ", « énergie » et « temps » constituent-ils les titres des découpes du texte qui retracent les points phares de la destinée d'Heisenberg de 1922 à 1945 ; la partie " positions » (LP : 9-56) se structure en quatre séquences : « Position 1 : Helgoland » (11-19) ; « Position 2 : hors de la demeure, sur un champ de ruines » (21-32); «Position 3 : dans la chambre à brouillard » (33-41) et «Position 4: entre le possible et le réel» (43-56). L'existence du savant s'y trouve envisagée sous le même prisme qu'une particule dans une chambre à brouillard ${ }^{14}$, ce dispositif destiné à observer la trajectoire des électrons. Dans l'ensemble du récit biographique, le lecteur ne découvrira que des positions, c'est-à-dire des moments forts et des états d'âme, nullement une destinée prévisible ou logique dans le chaos du siècle. C'est ainsi que les savoirs métabolisent le romanesque biofictionnel.

Dans les chapitres suivants qui sont découpés en parties non titrées et qui dépeignent autant des moments d'intimité que des succès professionnels, la figure ambivalente du savant de génie se prête tout aussi parfaitement à cette exploration. Collaborateur surdoué de Borm et de Bohr, qui vit «avec l'espoir, déraisonnable et magnifique, de parvenir un jour à la description objective du fond secret des choses » (LP: 46), le physicien émet l'hypothèse vertigineuse dès 1923 que «ce qui compose la substance du monde n'est pas matériel » (18). Il jette les bases théoriques de la mécanique matricielle, parallèlement aux recherches de Schrödinger sur la mécanique ondulatoire, en affirmant que ce qui est observable au niveau microscopique équivaudrait à ce qui a lieu aussi au niveau macroscopique. Couronné à l'âge de trente et un ans du prix Nobel de physique en 1932, Heisenberg accepte, sous le régime nazi d'être l'un des responsables du programme d'armement nucléaire, sans pour autant afficher jamais une quelconque sympathie pour Adolf Hitler. Bien que le projet Uranium, rival du projet américain Manhattan qui devait 
en 1945 doter les États-Unis de la première bombe atomique, n'aboutisse pas faute de moyens financiers à l'élaboration d'un explosif nucléaire allemand, la compromission morale du scientifique est flagrante et indéniable. Cependant, Ferrari dans son portrait n'a nullement le dessein de percer l'énigme de ce parcours singulier pendant la période la plus sombre de l'histoire allemande : il est bien plutôt intéressé par la dissection d'une âme humaine, grise comme elles le sont toutes, celle d'un homme à qui, selon lui, il fut donné de « regarder par-dessus l'épaule de Dieu » (11) et qui s'est finalement compromis par une collaboration avec le Mal. Le mystère autour de cette personnalité demeure car, alors que tous les savants et universitaires juifs (tel Einstein) avaient fui ou étaient persécutés, et que les plus éminents d'entre eux avaient reçu des invitations à émigrer (69), l'orgueilleux Heisenberg est délibérément demeuré en Allemagne afin d'œuvrer au sein du III Reich à la création d'« îlots de stabilité » (69) pour l'après-guerre, selon l'expression de Max Planck qui l'en aurait convaincu en 1941. Et, même si, après 1945, Heisenberg a activement participé à la reconstruction de l'université allemande, il reste sous la plume bienveillante de Ferrari ${ }^{15}$ un réel complice par passivité des tenants de la physique aryenne $(79,83)$. La phrase se fait longue et ondulante pour faire entendre les reproches car la responsabilité des scientifiques est une question clairement posée dans le roman, en particulier au moment de la destruction de Hiroshima (108-110), cependant que Ferrari utilise à plusieurs reprises le terme de péché $(83,141)$, autrement dit de faute ou de transgression de la loi divine, qui ne peuvent pas être prévues au moment où le phénomène est découvert, et qui conduit à la mort de l'âme. L'empathie est rarement réprimée à l'adresse du savant allemand, «le fidèle, le brillant, le sensible Werner Heisenberg " (85), car l'écrivain opte pour une interprétation positive des choix de l'«impassible et joyeux» (53) physicien; il ne manque pas de rappeler en détail le contexte historique menaçant et brutal, chaotique pour un être aussi sensible, mélomane et précocement intelligent (160). Interrogeant la guerre, ou plus précisément les choix qui s'offrent aux hommes en période de crise civilisationnelle, il dépeint néanmoins «ce regard plein de terreur et de prières, ou plein de dégoût, de défi, de renoncement, qu'on reconnaît à chaque fois, quoi qu'il exprime, parce que c'est le regard du siècle » (72-73), retenant à demi sa tentation du pathos dans certaines scènes.

En faisant d'Heisenberg un poète (LP : 19), Ferrari le rapproche du prêtre (148). Tous deux côtoient la beauté du monde, qui est l'essence de l'existence, et sont sommés de dire avec un appareillage inadéquat cet indicible, versant irrémédiablement dans la spiritualité. "Comme nous appartenons aux lacs et aux montagnes, nous appartenons au crime" (129). À l'instar de tous les grands physiciens, conscients «que les choses n'ont pas de fond, et que le principe instaure entre elles et nous une limite infranchissable, un isthme au-delà duquel s'étend le néant ineffable » (46), Heisenberg dès l'ouverture apparaitt rêveur (si ce n'est naï) et épris d'infini et d'absolu. Son mysticisme naît autant de ses découvertes qui le rapprochent de Dieu que de son esthétisation de la réalité, de son inébranlable "foi en la beauté » (13) du monde ${ }^{16}$ et de l'art, et singulièrement de la musique, puisque la chaconne de Bach en ré mineur jouée par un violon soliste sert de fond sonore mélancolique à la narration $(18,30,39,62,151)$. Le versant biographique est par conséquent loin d'imposer seul sa teneur à ce roman polymorphe. 


\section{"Le roman, c'est la forme » ou la question de l'hybridité textuelle}

11 Mû par une ambition littéraire et philosophique forte, Ferrari tisse des liens entre le passé et le présent au cœur d'une fiction érudite, composite ${ }^{17}$ : à la biographie, il amalgame des fils narratifs de natures différentes. Il y a d'abord une part autofictionnelle puisque le narrateur, anonyme, possède des velléités d'écriture, évoquant « un roman que je vais bientôt écrire ", cependant que sa réticence à poursuivre ce projet prime ( $L P$ : 68-69). Finalement, il note, dépassé par l'absolu de la littérature auquel il souhaiterait tout mesurer: "Je pense de moins en moins au roman que je voulais écrire » (81). Les dernières notations concernant ce dessein dépeignent un Je désillusionné et amer ( 95 , 147). Dans la clausule, le Je se rapprochera pourtant du regard contemplatif du Vous dans une sorte d'instant de communion où il percevra à son tour, malgré l'omniprésence de la mort sur la terre des hommes, la beauté incomparable et fascinante du monde (161). Ce double fictif de l'auteur est une subjectivité qui, tout en retraçant quelques épisodes de sa propre existence, ordonne le texte et ses allers-retours entre l'histoire et l'actualité en s'adressant à Heisenberg : "[...] en vérité, ce sont les traces de votre parcours que je suis, jusqu’à Berlin, en $1933 »$ (69).

12 Il est notable que ses premiers souvenirs datent de 1989, année de la chute du mur de Berlin signant la fin de la guerre froide entamée quelque quarante années auparavant. Le narrateur passe une épreuve orale d'épistémologie et, face à sa séduisante examinatrice, il sèche lamentablement devant la théorie des quanta ( $L P: 27-28)$. Il s'impose en héritier en raison du thème mais également de la date de son examen : le présent qu'il vit a été noué il y a bien longtemps par les contemporains d'Heisenberg, ce qu'il notifie ainsi, s'adressant au physicien dans une subtile anticipation métanarrative ${ }^{18}$.

[...] vous ne pouvez pas savoir que quelque chose vient de commencer pour

l'Allemagne qui ne s'achèvera qu'en ce mois de novembre 1989 durant lequel je regarde incrédule [...] ce que vous auriez tant voulu voir et ne verrez pas, la chute du mur que je croyais éternel et la fin du seul monde que j'avais connu jusqu'ici. (LP :54)

13 Même si l'on sait Ferrari passionné par les sciences exactes depuis ses études de philosophie, sa biographie d'Heisenberg ne se greffe pas à un texte autobiographique. Certes, dans les passages dédiés aux années 1989-2009, l'on reconnaît aisément des motifs présents antérieurement sous sa plume, parmi lesquels se trouvent les reliefs corses auxquels l'auteur, né à Paris de parents natifs de l'île, ne manque jamais de rendre hommage dans ses romans ${ }^{19}$. C'est du reste dans les paragraphes descriptifs qui disent les paysages et le tempérament corses que l'aspect visuel ${ }^{20}$ de son écriture est le plus net. Le matériau autobiographique, attaches affectives comprises, est tenu à distance par la voix narrative grâce à un dépli solennel d'images qui sied à la période des grands règlements de compte entre mouvements nationalistes - la Corse de 1995 est violente et meurtrie (LP : 67-68 ; 80-81; 95-97) - et au deuil de la mère, qui sont deux confrontations brutales et personnelles avec la mort.

14 L'Arabie est l'autre territoire du Je qui lui offre de donner sa vision du monde des pétrodollars où la passion économique domine, supplantant l'essentiel qu'est le respect de la dignité humaine. Le narrateur dessine les contours actuels des pays du golfe 
Persique dont l'environnement a subi des transformations radicales à une vitesse vertigineuse depuis la découverte du pétrole dans le second demi-siècle.

En moins de quarante ans, sur le sable du désert, au bord de la mer brûlante où plongeaient tout l'été de misérables pêcheurs de perles, le pétrole a fécondé la terre aride, il en a fait surgir des tours de verre, de marbre et d'acier qui s'élancent toujours plus haut dans les fournaises poussiéreuses du ciel. (LP : 146)

Suivant un même décalage entre autobiographie et fiction, Ferrari choisit d'évoquer l'émirat de Dubaï ${ }^{21}$ et non celui d'Abu Dhabi où il a travaillé pendant deux ans au lycée français ${ }^{22}$ : le Je peint une mégalopole d'une modernité totale, avec force métaphores végétales et animales ("plante carnivore» et "bête à l'agonie» (LP: 151-152) et finalement humaines, sur un ton aux élans apocalyptiques à la mesure du désarroi que l'auteur lui-même a ressenti sur place et que son narrateur traduit ${ }^{23}$.

[...] je suis assis, pour la dernière fois, dans un taxi roulant vers l'aéroport, sur la Sheikh Zayed Road, un soir de septembre 2009 [...] La plus haute tour du monde se dresse dans la nuit comme la flèche d'une cathédrale énorme - non vous avez raison, elle se dresse plutôt comme une monstrueuse plante carnivore, enfonçant profondément ses racines dans le sable du désert [...] Il ne lui manque rien, pas même le sang, car toute la ville est cimentée par le sang dont elle est nourrie et se nourrit encore. Le sang indien, le sang pakistanais, le sang du Bangladesh, du Népal et du Sri Lanka, tout ce sang anonyme qui court inlassablement dans ses veines d'acier et la fait proliférer. (LP: 152)

Reste le portrait d'une décadence annoncée, à la fois écologique (la pollution engendrée par l'urbanisation rapide et massive de cet émirat est démesurée) et humaine : comme le chauffeur de taxi népalais qui, tétanisé de peur, conduit le narrateur vers l'aéroport, les travailleurs émigrés, souvent exploités, affluent de toute l'Asie vers ce nouvel Eldorado: $90 \%$ des 1,6 million d'habitants de la capitale sont des étrangers qui parfois vivent dans des conditions à la limite de l'insalubrité (LP: 146). Ferrari met en relation le cycle infernal de la pauvreté, l'outrance visuelle et urbaine de ce pôle consumériste, avec le constat scientifique selon lequel les réserves pétrolières et gazières s'amenuisent de décennie en décennie et que, par conséquent, le déclin de l'émirat est économiquement prévisible :

Une lente paralysie gagne tous les organes vitaux de la ville qui halète comme une bête à l'agonie. À peine née, elle va déjà mourir. Nulle part ailleurs, le processus aveugle de la vie et de la mort n'avait manifesté son incontrôlable puissance avec une telle pureté, jamais il ne s'était déroulé à une rapidité si effroyable. Vous n'en seriez sans doute pas étonné. Vous savez qu'en un temps très court, une énergie presque infinie peut jaillir du néant avant d'y retourner. (LP : 152-153)

L'autofiction devient une composante supplétive de la compréhension historiographique et, l'engagement de l'artiste dont le vade-mecum semble tenir en ces quelques mots: Vanitas vanitatis, est tout entier dans ce travail sur l'histoire ${ }^{24}$. Dans la quatrième partie du Principe, intitulée "temps ", le narrateur entreprend un flash-back le menant à l'époque médiévale musulmane, confirmant son désir de proposer grâce à son art du récit une explication $\mathrm{du}$ monde: gouttelettes dans une chambre à brouillard, citations jüngeriennes ou haltes niffariennes en font de même par leur hétérogénéité foncière.

Du sultanat d'Oman aux rives du golfe dont les Perses et les Arabes se disputent encore l'honneur d'être les héros éponymes, à travers les sables qui furent la demeure séculaire de Bédouins pour lesquels nulle beauté, si ce n'est celle de Dieu, ne surpassait la beauté de la poésie, il reste sans doute encore des hommes pour s'éblouir des vers incomparables qu'Al Mutanabbi composa il y a plus de mille ans : Les chevaux, le désert et la nuit me connaissent, 
Et l'arc et l'épée, le papier et la plume

Mais plus personne ne peut en reprendre à son compte l'orgueil incommensurable. Ils sont devenus les reliques muettes d'un monde soudainement disparu, un trésor antique, étrange et vénéré, qui luit désormais d'un éclat incompréhensible dans le sanctuaire d'un temple vide. (LP: 145)

Les vers d'al-Mutanabbi $\bar{i}^{25}$, orgueilleux à l'éloquence superbe, habitué aux prouesses poétiques, concrétisent ce détour explicatif vers le passé : le Je remarque que les mots qui firent la fierté du poète du $\mathrm{x}^{\mathrm{e}}$ siècle et l'ont conduit à la mort ${ }^{26}$, n'ont plus de pertinence dans un présent en perte de sens ( $L P: 154)$. Ferrari suggère qu'une semblable tâche incombe au poète et au physicien, celle de "dépasser infiniment les ressources de la langue pour dire ce qui ne peut l'être et pour décrire aussi précisément que possible tous les ordres d'une réalité hypothétique, multiple, indescriptible» (95). Il étaye cette suggestion en s'appuyant sur l'existence et la pensée d'un célèbre auteur allemand puisqu'il a longuement séjourné en Allemagne pendant la préparation du Principe, dévorant notamment l'œuvre de Jünger. En conséquence, le destin d'Heisenberg se dévide parallèlement à des étapes de la non moins riche destinée d'Ernst Jünger ${ }^{27}$ (1895-1998), controversé en raison de sa jeunesse militariste, mais emblématique des tourments de son époque par sa vie et ses écrits, notamment $L a$ Guerre comme expérience intérieure ${ }^{28}$ qui se composent de souvenirs de combats, de réflexions philosophiques et politiques sur la guerre. Ferrari marque son admiration pour Jünger et pour les écrivains allemands dont la dimension métaphysique des œuvres n'est pas plus à démontrer que la puissance spirituelle qui s'en dégage; il bâtit à l'aide d'événements phares de l'existence du soldat écrivain un double de son protagoniste accomplissant un destin en tout point différent puisque, même s'il fut officier de l'administration militaire d'occupation à Paris en 1941, Jünger a su se tenir éloigné de la vie politique à partir de l'accession des nazis au pouvoir. Exempt de toute compromission avec le III ${ }^{e}$ Reich (90) grâce à des prises de position fortes (il dénonce la barbarie nazie en 1939 dans un roman allégorique intitulé Sur les falaises de marbre ${ }^{29}$ ), l'auteur d'Orages d'acier (qui raconte ses souvenirs de soldats durant la Première Guerre) figure un autre possible pour dire le $\mathrm{xx}^{\mathrm{e}}$ siècle, pour s'interroger sur l'être et le néant, et surtout un contrepoint pour encore une fois témoigner que toute action humaine (guerre comprise) se compose de bien et de mal. Selon Jünger, tout conflit ne saurait indiquer autre chose que l'amour de Dieu à l'égard de ses créatures et Ferrari l'explique par l'exemple. À l'instar de tous les personnages du Principe, Jünger apparaît dans plusieurs mini-portraits : d'abord, juste après le souvenir flou du père d'Heisenberg en uniforme quittant sa maisonnée pour les tranchées de la Grande Guerre lorsque le lieutenant Jünger «les larmes aux yeux [...] écrit dans son carnet: mais quand donc finira - quand donc finira cette guerre de merde ?» (13-15). Cette vision permet au Je de noter, en accord avec Jünger, que l'essence du combat, sa rage, est une pulsion des plus archaïques. Jünger réapparait ensuite au cœur du second conflit mondial, dans une esquisse qui exprime tout le contraste de sa sensibilité médiumnique, teintée d'une lucidité désabusée autant qu'éprise d'absolu (76). Parce qu'elle est révélatrice des tréfonds de l'âme humaine aux prises avec la mort imminente, la prose de Jünger, née d'un regard pertinent posé sur un monde en voie de métamorphose, passionne Ferrari : le rapport au monde de l'auteur allemand est entièrement structuré par la leçon d'Héraclite - la référence à la sagesse héraclitéenne est récurrente dans l'œuvre de Ferrari - et par la prophétie nietzschéenne, cet amor fati, qui impose de ne pas vouloir autre chose que ce qui est. Les conflits que Jünger a traversés lui ont enseigné qu'eux seuls ramènent l'existence à sa plus élémentaire pulsation, celle du vouloir-vivre porté à son acmé dans 
sa confrontation avec la mort (92). Aussi, est-ce à parts égales que les champs sémantiques et lexicaux de la beauté et de l'horreur envahissent le tissu narratif : « il y a un vertige de l'horreur plus puissant, peut-être, que celui de la beauté » (14). Débordant d'élans emphatiques sur la grandeur de la connaissance et les ouvertures infinies que procure la physique atomique, accumulant les gros plans sur la chair humaine torturée par les bombes $(14,94,108-110)$, multipliant les sentences méditatives sur la finitude humaine - et sur ce que vaut un homme, sur ce que sont sa dignité, sa faiblesse et sa souffrance fondamentales -, sans s'interdire quelques pointes humoristiques ${ }^{30}$, le roman de Ferrari vaut autant pour l'hybridité foncière de sa texture que de ses motifs.

\section{Physique et métaphysique}

19 Aujourd'hui, la fiction biographique telle que l'appréhende avec Le Principe Jérôme Ferrari, mêlant connaissances scientifiques, postures autofictionnelles et narration fictionnelle, est plébiscitée dans le monde littéraire en langue française - la consécration nationale d'auteurs comme Laurent Binet, Patrick Deville, et de façon plus évidente et internationale celle de Houellebecq, sont autant de signes, parmi d'autres, de ce plébiscite -, d'autant qu'elle porte en son sein une question spirituelle. La forme vocative, qui s'impose dans l'ensemble du récit à l'exception d'un épisode de la partie « énergies » ( $L P: 105-142$ ), possède une certaine emphase, un lyrisme qui sied à la prose de Ferrari dans ce dialogue avec le passé basé sur un décalage générationnel et civilisationnel entre Heisenberg et le Je. Ce Vous lancé vers l'outre-tombe offre au narrateur de s'approprier les pensées du héros, de rester au plus près de ses sentiments et de ses sensations, comme s'il s'agissait de conjurer la biographie. La reprise d'une narration en forme de longue adresse $^{31}$, expérimentée avec Un dieu un animal (2009), est indissociable d'un projet stylistique qui révèle le désir d'exprimer, grâce à une tonalité solennelle, l'humanité dans ce qu'elle a de bon, de beau et de bienveillant mais aussi de cruel. Lors du gros plan (111-142) établi sur le huis clos rassemblant dix des plus éminents savants allemands qui eut lieu en Angleterre, dans le cottage de Farm Hall pendant l'été 1945, le jeu entre le Vous et le Je narratif s'estompe. La parole est confiée aux scientifiques emprisonnés par l'armée britannique : Von Laue, Hahn, Gerlach, Harteck, Von Weizsächer, Wirtz, Diebner, Bagge et Korsching et Heisenberg qui incarnent la capitulation des universitaires, restés aveugles à la politique de leur nation sous le III ${ }^{\mathrm{e}}$ Reich. Responsables ${ }^{32}$, sont-ils coupables ? Tous tentent de se dédouaner individuellement mais ressentent la culpabilité nationale dans un Nous maladroit. Hiroshima et Nagasaki viennent tout juste d'être bombardées et le Je les accable pour leurs réactions - sidération, envie, incrédulité, horreur, soulagement, curiosité, déception, amertume se mélangent en eux (115) - avant de les absoudre : «ils vivent dans un monde qui n'existe pas » (129), explique le narrateur, sensible au fait que ces savants plaçaient si haut la science et son objectif supérieur de vérité des choses qu'il ne peut imaginer qu'ils aient pu être corrompus par l'idéologie nazie auxquels du reste ils s'intéressaient peu ${ }^{33}$ :

Ils voulaient comprendre, regarder un instant par-dessus l'épaule de Dieu.

La beauté de leur projet leur semblait la plus haute qu'on pût concevoir.

Ils étaient arrivés là où le langage a ses limites, ils avaient exploré un domaine si radicalement étrange qu'on ne peut l'évoquer que par métaphores ou dans l'abstraction d'une parole mathématique [...] ils devaient sans cesse réinventer ce que signifie « comprendre ».

Les connaissances qu'ils vénéraient ont servi à mettre au point une arme si 
puissante qu'elle n'est plus une arme, mais une figure sacrée de l'apocalypse.

Ils en ont tous été les oracles et les esclaves. (126) émet ses propres doutes d'homme et de romancier: à la faveur d'une pensée rhizomatique, il construit son intrigue à l'aune des savoirs quantiques, métaphorisant des questions et des paradoxes soulevés par les scientifiques parmi lesquels Heisenberg, luimême auteur d'un ouvrage intitulé Physique et philosophie ([1961] 2000) mais aussi par les intellectuels, artistes et penseurs. En fait, les scientifiques comme Heisenberg ou Bohr - Einstein n'avait pas de semblables préoccupations - avaient le désir de passer par les mots pour partager le sens physique et ils n'avaient donc de recours possible pour exprimer ce sens que dans les métaphores.

21

insi, afin de parcourir l'histoire des connaissances depuis le Timée platonicien jusqu'aux plus récentes théories de la matière, Le Principe s'inspire-t-il d'un intertexte rhizomatique, constitué par les épigraphes d'Héraclite, philosophe grec présocratique du $\mathrm{IV}^{\mathrm{e}}$ siècle avant J.-C. dont la pensée instille tout le roman, et de Niffari ${ }^{34}$, poète mystique musulman du $\mathrm{x}^{\mathrm{e}}$ siècle influencé par la spiritualité persane à thèmes soufis ${ }^{35}(L P: 7,93)$ ainsi que des vers du poète arabe al-Mutanabbī (145) et des nombreuses références à Jünger. La guirlande de personnages qui scintillent à travers les âges ou pour emprunter l'image des physiciens, l'ensemble des gouttelettes disséminées dans une chambre à brouillard qui matérialise des électrons que l'esprit humain a tendance à relier dans une illusion de trajectoire, fait écho aux haltes de Niffari, ces courts poèmes spirituels, énigmatiques et rétifs à toute réduction rationnelle, qui constituent chacun une voie singulière menant à Dieu. Ces références elliptiques en arborescence ne sont pas à considérer comme des étapes sur un chemin, celui d'une quête de l'absolu sur lequel a marché sa vie durant Niffari, mais bien plutôt, selon l'intuition soufie, comme des états fulgurants, transcendants, où saisir l'être dans sa totalité et son unicité. Aussi, chaque brin du rhizome qui présente à la fois des points communs et des divergences partage-t-il avec les autres un identique enthousiasme mystique ${ }^{36}$. Que l'ordre du monde soit imposé par une instance transcendante est une possibilité admise par le narrateur qui relève dans des hypotextes littéraires de tradition occidentale ou perse et arabo-musulmane un point commun : la notion d'union mystique ${ }^{37}$. Selon la recherche islamologique, le soufi est en effet un individu qui doit atteindre la "science de l'unicité de dieu ${ }^{38}$ » qui serait sa réalisation spirituelle totale. Ce qui caractérise cette pensée musulmane ésotérique et déroutante, c'est précisément l'union des contraires, du singulier et du pluriel comme du proche et du lointain, non leur disparition au sein d'une unité mystérieuse. C'est ce qu'au cœur de la matière, Heisenberg a pu découvrir, à savoir que l'infiniment grand rejoint l'infiniment petit. Ce qui fascine Ferrari et le touche profondément dans cette mystique qu'il a découverte lors de son séjour en Algérie, c'est sa façon d'englober le double aspect contradictoire de la vie et des choses, sans le résoudre; il y cerne quant à lui la seule vérité possible, l'unique possibilité de tenir compte de la violence et du nihilisme des extrémismes politiques. De fait, le rôle des références à cette mystique est important dans l'économie du roman puisque le Je suggère que le dessein des soufis et des savants est face au néant d'exprimer le néant et face à la beauté, d'exprimer la beauté, néant et beauté n'étant pas incompatibles avec l'existence de Dieu. « La promesse d'un destin parodiant le hasard dont l'accomplissement serait tout à la fois un triomphe, une chute et une malédiction » (142). D'ailleurs, les thématiques qui globalement hantent les philosophes et écrivains cités sont également celles qui se trouvent au centre de l'écriture et de la 
réflexion ferrarienne : la perte, la chute, la rupture, le néant ${ }^{39}$. Pour Ibn 'Arabi, qui se situe intellectuellement dans la lignée de Hallâj, la voie mystique n'est ni rationnelle ni irrationnelle, l'esprit s'échappe des limites de la matière et contrairement à la philosophie, elle se situe hors du domaine de la raison. En évoquant la foi d'Heisenberg, cette confiance irraisonnée en Dieu, en la beauté du monde et en la science, le Je suscitant ces rapprochements, s'interroge pour lui et ses semblables sur la nature humaine. «Cette vie elle aussi deviendra floue, elle rejoindra les fantômes d'autres vies, perdues dans les limbes, entre le possible et le réel auxquelles rien ne la relie » (158). Le regard du savant s'enracine dans l'intuition du métaphysicien et Ferrari, qui s'affirme comme agnostique mais affiche un net penchant au mysticisme, oriente son œuvre vers d'autres interrogations. Si le discours éthique (philosophique et idéologique) l'imprègne, le roman s'éloigne d'une quelconque tendance moralisatrice, malgré une vision cyclique de l'aventure civilisationnelle. Du reste, le Je désabusé redit après Heisenberg sa croyance en la persistance de la beauté au-delà du Mal. « Il existe un lieu où l'amour de Dieu ne ment pas » $(148,153)$.

Avec Le Principe, qui repose sur un lien fort entre une interprétation de la physique quantique et l'écriture littéraire, Ferrari poursuit sa quête d'un romanesque protéiforme, apte à dire l'irisation de notre monde. Il élabore un dialogue inattendu entre le passé et le présent, l'histoire des sciences et l'art poétique, le romanesque actuel et les grands traumatismes historiques $\mathrm{du} \mathrm{xx}^{\mathrm{e}}$ siècle, la philosophie et le mysticisme. De fait, il se trouve dans le questionnement épistémologique qui transcende cette œuvre sous la forme d'un devoir de mémoire, un regard original sur les fondations de notre modernité.

\section{BIBLIOGRAPHIE}

Binet, Laurent, 2010, HHhH, Paris, Grasset \& Fasquelle, 2010.

Binet, Laurent, 2011, « Le merveilleux réel », Le Débat, n 165, p. 80-85.

Caminade, Emmanuelle, 2009, « Entretien avec Jérôme Ferrari », L’Or des livres, [En ligne], http://

l-or-des-livres-blog-de-critique-litteraire.over-blog.com/article-32118605.html, consulté le

5 février 2018.

Ferrari, Jérôme, 2002, Aleph zéro, Ajaccio, Albiana.

Ferrari, Jérôme, 2009, Un dieu un animal, Arles, Actes Sud, coll. « Babel ».

Ferrari, Jérôme, 2012, Le Sermon sur la chute de Rome, Arles, Actes Sud, coll. « Babel ».

Ferrari, Jérôme, 2015, Le Principe, Arles, Actes Sud, coll. « Domaine français ».

Forest, Philippe, 2013, Le Chat de Schrödinger, Paris, Gallimard. 
Heisenberg, Werner, [1961] 2000, Physique et philosophie. La science moderne en révolution, Paris, Albin Michel.

Hofstadter, Douglas, 2008, Gödel, Escher, Bach : Les Brins d'une guirlande éternelle, trad. de l'anglais par Jacqueline Henry et Robert M. French, Paris, Dunod.

Houellebecq, Michel, [1998] 2001, Les Particules élémentaires, Paris, Flammarion, coll. «J'ai lu ». Jünger, Ernst, 2008, La Guerre comme expérience intérieure, traduit par François Poncet, Paris, Christian Bourgois.

Niffari, Muhammad, 2007, Les Haltes, trad. de l'arabe, présenté et calligraphié par Sami Ali, Arles, Actes Sud.

Rio, Michel, 1992, Le Principe d'incertitude, Paris, Seuil.

Schiff, Daniel, 1982, La Ligne de Sceaux, Paris, Minuit.

Schmidt, Jean-Jacques, 1994, al-Mutanabbì. La Solitude d'un homme (choix, traduction et présentation des textes), Paris, Orphée, La Différence.

Toussaint, Jean-Philippe, 1987, Monsieur, Paris, Minuit.

Viart, Dominique, 2008, " Mémoire et enquête : la seconde guerre mondiale », dans D. Viart et B. Vercier (dir.), La Littérature française au présent : héritage, modernité, mutations, Paris, Bordas, p. 146-171.

\section{NOTES}

1. Agrégé de philosophie et titulaire d'un DEA d'ethnologie, Ferrari, né en 1968, est enseignant dans le secondaire ; il est également traducteur et, depuis 2001, il a traduit du corse trois œuvres du poète Marcu Biancarelli.

2. Traduit rapidement après sa sortie en anglais, allemand, italien et espagnol, le roman a remporté un franc succès international.

3. Abrégé en $L P$ dans le reste de l'article. Seule la pagination est mentionnée entre parenthèses lorsque plusieurs références à cette œuvre se suivent dans un même paragrpahe.

4. Les sermons furent prononcés par saint Augustin en 410 à Hippone dans le contexte des avancées des Barbares de l'Est, juste après le sac de Rome par Alaric ; leur évocation, entre faillite des systèmes de croyance et perte des repères, structure Le Sermon sur la chute de Rome rappelant que nul empire n'est immortel et qu'à la gloire et à l'opulence succède inéluctablement la fin douloureuse. Dans La Cité de Dieu, saint Augustin écrit en substance : «Le monde est comme un homme : il naît, il grandit et il meurt. "

5. Les romans ferrariens s'intègrent dans un corpus français d'œuvres fictionnelles contemporaines qui se saisissent de l'histoire coloniale et postcoloniale, y renouvelant les perspectives éthiques en s'attachant aux figures des victimes, des bourreaux, des héros.

6. Pensons à Yannick Haenel (Jan Karski, Paris, Gallimard, 2009), Laurent Mauvignier (Des Hommes, Paris, Minuit, 2009) ou Alexis Jenni (L'Art français de la guerre, Paris, Gallimard, 2011).

7. Sous la plume de l'écrivain, s'épanouissent d'autres topiques: la Corse, la philosophie et ses grands représentants de saint Augustin à Heidegger, l'oscillation entre la gloire et les empires des hommes et leur irrémédiable chute, façon d'autrement traduire la locution latine Sic transit gloria mundi.

8. En allemand Der Teil und das Ganze, l'ouvrage est paru en France en 1972.

9. D'autres photographies d'Heisenberg à différents âges et dans différents lieux sont décrites dans le roman (Ferrari $2015: 44,53,56,148$, etc.). 
10. La physique quantique s'astreint alors à résoudre des problèmes que la physique classique échouait à expliquer comme les caractéristiques expérimentales des trous noirs, l'effet photoélectrique, etc.

11. Les physiciens parlent de principe d'indétermination, selon la traduction allemande. Ferrari note cette indécision du lexique (Ferrari $2015: 48$ ).

12. En fait, notre compréhension du monde est structurée par les concepts linguistiques qui ne sont pas applicables à cette échelle. Et pour Ferrari, la capacité de l'homme à connaitre le statut de la réalité s'étiole dans sa prétention vaine à tenir des discours qui en diraient le sens.

13. La faillite du langage est ainsi plusieurs fois reformulée : "vous saviez qu'il faudrait en venir à la cruelle nécessité d'exprimer, comme le font les poètes, ce qui ne peut l'être et devrait être tu [...] faire dire aux mots ce qui ne peut être dit mais doit cependant l'être" (Ferrari 2015 : 47-48).

14. La chambre à brouillard est une enceinte, étanche ou non, utilisée comme détecteur de particules: elle permet de voir sous la forme de traînées de condensation le passage des particules nucléaires dans la matière. Ferrari cite les premières créées vers 1910 : les chambres de Wilson (Ferrari $2015: 33$ ).

15. Il ne revient pas au romancier d'adhérer ou non au comportement d'Heisenberg, mais plutôt d'adopter une position intellectuelle éclairante. Les références émises par Heisenberg au mouvement d'étudiants anti-nazis de la Rose Blanche (Ferrari 2015 : 128) suggèrent tout de même que le romancier opte pour un physicien humaniste et sincère à la recherche de la vérité.

16. Le narrateur décrit l'exceptionnelle beauté des paysages allemands, les vallées des Alpes bavaroises de la Walchensee ou l'archipel d'Helgoland (qui, en bas allemand, signifie "terre sacrée ») situé dans le sud-est de la mer du Nord, dont la contemplation constitua des haltes vivifiantes (des moments de grâce) pour Heisenberg répétant à l'envi (Ferrari 2015 : 161). Le physicien à l'âme géographique s'est rendu au Japon (Ferrari 2015 : 56).

17. L'expression choisie comme titre est du romancier Patrick Deville, lauréat du prix Femina en 2012 avec Peste \& Choléra, qui partage avec Ferrai un goût de l'hybridation et une passion pour la philosophie (qu'il a enseignée), l'épistémologie, l'histoire et le récit de vie.

18. Plus loin, il ajoute : « le monde qui s'efface tout entier sous vos yeux, je suis désolé de vous le dire, vous ne le reverrez jamais »; «Ce que la guerre a brisé ne sera pas réparé » (Ferrari 2015 : $62 ; 155)$.

19. Depuis 2015 et son retour du Golfe, Ferrari enseigne au lycée Giocante de Casabianca à Bastia.

20. C'était déjà le cas dans Le Sermon sur la chute de Rome.

21. Jamais nommée, la mégalopole est reconnaissable notamment à la tour Khalifa, «la plus haute tour du monde » (Ferrari $2015: 151)$.

22. Le lycée est comme un heureux hasard nommé Louis-Massignon. Pour mémoire, rappelons que Massignon (1883-1962) est un éminent islamologue qui permit la diffusion en Europe de la pensée du mystique musulman Mansur Hallâj, grâce à ses nombreux travaux de recherche, et notamment une monographie érudite intitulée La Passion de Hallâj, martyr mystique de l'Islam (rééditée par les éditions Gallimard en 2010). Parmi ses étudiants figure Henry Corbin, spécialiste, quant à lui, du mystique hispano-arabe Ibn 'Arabī. Ferrari a auparavant enseigné quatre années en Algérie (au lycée Alexandre-Dumas d'Alger) et c'est pendant ce séjour qu'il a découvert la mystique soufie, précisément grâce aux poèmes d'Hallâj et ceux d'Ibn 'Arabī (1165-1240).

23. En contrepoint, on lira la longue citation d'Heisenberg qui compare l'évolution de la technique à un processus organique, «un événement biologique » (Ferrari 2015: 151). Le narrateur file la métaphore sur plusieurs pages : «D'étranges excroissances de nos organes ont inexorablement envahi le monde. Elles l'ont transformé. La chair s'est faite verre et métal » (Ferrari 2015 : 153). 
24. Les œuvres ferrariennes sont globalement traversées et travaillées par des réflexions et des questionnements sur l'effondrement des empires, la faillite des systèmes de croyance et la perte douloureuse des repères laissés chez les individus et dans le corps social. À l'instar du Principe, certaines d'entre elles, Un Dieu un animal et Où j'ai laissé mon âme par exemple, s'intègrent dans la littérature des guerres françaises, commentées par Dominique Viart dans le second chapitre de la section « Écrire l'histoire » (2008: 146-171).

25. al-Mutanabbī (915-965) est un poète arabe qui vécut à l'époque de la domination persane et turque de l'Empire arabo-musulman ; génie poétique, symbole de l'arabité, il est demeuré célèbre pour son verbe virtuose et acerbe ainsi que pour sa peinture désabusée, d'une lucidité sans complaisance, de ses contemporains au moment de la décadence de l'Empire.

26. Habitué à louer les seigneurs entre Damas et Bagdad, le poète provoqua par ses dires l'ire d'un clan et mourut dans un guet-apens, encouragé par ce vers, le plus célèbre de ses nombreuses compositions, cité en guise de provocation par celui qui le mettra à mort. On consultera avec profit Schmidt (1994).

27. Les deux hommes se rencontrent à Munich en 1953 lors d'une conférence (Ferrari 2015 : 147-150).

28. L'ouvrage La Guerre comme expérience intérieure, traduit par François Poncet, a été réédité par Christian Bourgois en 2008.

29. Ce roman est cité comme par inadvertance par le narrateur lorsqu'il rappelle un épisode terrible de l'existence de Jünger, le décès à 18 ans de son fils aîné, soldat sur le front aérien italien de 1944, et qu'il évoque : «un jeune garçon [...] les yeux grands ouverts tournés vers le ciel, sur les falaises de marbre que son père inconsolable a dressées pour lui comme un berceau » (Ferrari 2015 : 90).

30. La phrase, sinueuse et longue, parfois se gonfle d'ironie et devient joueuse (Ferrari 2015 : 36 , 108) : «[...] le regard des physiciens ne sera jamais celui de Dieu. On ne dévoilera pas les plans du vieux, à peine peut-on espérer jeter furtivement un œil par-dessus son épaule » (Ferrari 2015 : 46).

31. Ferrari explique : «La narration à la deuxième personne fait partie du projet initial du roman, elle en est indissociable. Elle ouvrait les perspectives stylistiques dont j'avais besoin, un certain ton, solennel et liturgique, la possibilité d'exprimer immédiatement la bienveillance et la cruauté et elle résolvait (cela, je l'ai découvert en écrivant) le problème du passage en continu d'un personnage à l'autre » (dans Caminade 2009).

32. Bagge et Diebner furent les seuls membres du parti nazi.

33. Absoudre semble un terme religieux fort approprié pour évoquer le pardon du narrateur accordé à ces hommes qui, selon lui, ont commis un péché, péché d'orgueil au premier chef qui eut pour conséquence incontrôlable un déferlement d'actions violentes et meurtrières.

34. Demeurées dans l'oubli pendant plus d'un siècle et demi, les deux œuvres majeures de Muhammad Al-Niffari (?-965), et en particulier Les Haltes, ont été redécouvertes par Ibn 'Arabi. Ferrari en cite cet extrait: «Et Il m'a dit: Entre la parole et le silence, il y a un isthme où se trouvent la tombe de la raison et la tombe des choses» (Ferrari $2015: 7)$. M. Niffari, Les Haltes (trad. de l'arabe, présenté et calligraphié par Sami Ali), Arles, Actes Sud, 2007.

35. Dans Aleph zéro et Un dieu un animal (placé sous l'épigraphe du poète persan El Hallâj (858-922), l'intérêt de l'auteur pour la mystique soufie (elle constitue le cœur ésotérique de la tradition islamique) s'était déjà manifesté sous forme d'interrogation sur la transcendance.

36. Pour comprendre la démarche artistique de Ferrari, le lecteur lira avec profit Hofstadter (2008). Cet ouvrage qui a obtenu le prix Pulitzer de l'essai en 1980 cerne la façon dont les réalisations du logicien Kurt Gödel, de l'artiste Maurits Cornelis Escher et du compositeur JeanSébastien Bach s'entrelacent, s'appellent ou s'excluent. À l'instar des personnalités citées par le narrateur de Ferrari, Gödel, Escher et Bach figurent des destinées apparemment indépendantes et projetées dans différentes directions par une essence centrale : au lecteur initié d'explorer 
ainsi les applications formelles à différentes échelles des théories des quantas et d'en saisir toute la portée épistémologique.

37. L'épigraphe d'Aleph zéro est citation d'Eugène Wigner, prix Nobel de physique en 1963 : "Chaque chose porte effectivement en elle son contraire mais la conscience qui s'en saisit la contraint à ne présenter qu'un seul de ses visages possibles. Personne n'a songé à la portée théologique d'une telle théorie qui réconcilie la toute puissance et la bonté de Dieu » (Ferrari $2002: 7)$.

38. Traduite en français par Massignon, la pensée hallâjienne comporte le souhait de renouer avec la pure origine du Coran et son essence verbale et lettrique; elle se présente comme traitant de la science de l'unité.

39. Sur la question de la sacralisation de la violence et sur l'impossibilité pour tout un chacun d'accepter les liens entre la beauté et le néant, l'amour et la souffrance, Ferrari s'est expliqué sur le site L'Or des livres (Caminade 2009). Il affirme par exemple : "C'est ce qui caractérise la vision mystique, l'union des contraires, non leur disparition, au sein d'une unité mystérieuse. Hallâj, qui joue un rôle très important dans le roman, explicite constamment cela dans ses magnifiques poèmes. Dans l'un d'eux, il parle d'un hôte qui accueille son invité avec une grande bienveillance et le fait exécuter au matin. J'ai la conviction intime que Hallâj a compris sa propre exécution comme l'expression la plus haute de l'amour de Dieu. L'étreinte d'un être qui nous dépasse infiniment ne peut que nous détruire. Je ne suis pas moi-même mystique mais c'est vraiment quelque chose que je respecte beaucoup. J'y vois une tentative désespérée et magnifique de voir le monde tel qu'il est et de préserver malgré tout la possibilité de l'amour. »

\section{RÉSUMÉS}

Avec Le Principe (2015), Jérôme Ferrari poursuit sa recherche d'un romanesque complexe, apte à intégrer l'histoire du $\mathrm{xx}^{\mathrm{e}}$ siècle et ses traumas : son roman interroge, entre savoirs et fiction, la contemporanéité à l'aune du passé et d'une réflexion sur la transcendance. Suivant les grandes étapes de l'existence du physicien allemand Heisenberg (1901-1976), le narrateur (dont les inflexions autofictionnelles transportent le lecteur de Corse dans les années 1990 jusqu'à Dubaï aujourd'hui) revient ainsi sur la montée du nazisme, l'explosion des bombes atomiques sur le Japon, les désastres de l'après-guerre. Son approche de la physique quantique qui, avec le principe d'incertitude énoncé par Heisenberg, stipule l'incapacité du langage à saisir la réalité, s'établit grâce à des liens avec la pensée mystique soufie et irradie toute la narration. L'hybridité $\mathrm{du}$ roman en fait une œuvre singulière, une interrogation sur les liens entre connaissance et éthique, entre savoir scientifique et mysticisme, rattachée à une perspective épistémologique.

With Le Principe, published in 2015, Jérôme Ferrari continues his research for a complex novelistic able to integrate the history of the past Century and its traumas: the narrator returns to the rise of Nazism, to the explosion of the atomic bomb in Hiroshima and looks back on the disasters of the postwar. Thanks to the autofictional part of the novel, the reader goes also to Corsica in the nineties to Dubaï in the year two thousand and ten. The novelist follows the major stapes of the life of Heisenberg (1901-1976), a famous physicist who developed the "uncertainty principle" which is the one of the most important results of twentieth Century physics. He writes a hybrid and singular novel which questions, between knowledges and fiction, our present time in terms of the historical past and a reflection on the transcendence. 
INDEX

Mots-clés : roman français contemporain, histoire, savoir, épistémologie, soufisme, autofiction

Keywords : contemporary French novel, history, knowledge, epistemology, sufism, autofiction

\section{AUTEUR}

ISABELLE BERNARD

Professeure associée à l'Université de Jordanie 